\title{
Automated Precursor Ion Exclusion During LC-MS/MS Data Acquisition for Optimal Ion Identification
}

\section{Zhongqi Zhang}

Process and Product Development, Amgen Inc., One Amgen Center Drive, Thousand Oaks, CA 91320, USA

\begin{abstract}
Liquid chromatography-tandem mass spectrometry (LC-MS/MS) is widely used for characterizing multiple samples of complex mixtures with similar compositions. This article addresses a data acquisition strategy for collecting a maximal number of unique, high-quality MS/MS during LC-MS/MS analysis of multiple samples. Based on the concept that a component only needs to be identified once when analyzing multiple samples with similar compositions, an automated intersample data-dependent acquisition strategy was developed. The strategy is based on precursor ion exclusion (PIE) and is implemented in MassAnalyzer in an automated fashion for Thermo Scientific (San Jose, CA, USA) mass spectrometers. In this method, MassAnalyzer submits one sample at a time to the sample queue. After data acquisition of each sample, MassAnalyzer automatically analyzes the data to generate a PIE list based on the MS/MS precursor ions, merges this list with the list generated from previous runs, adds the list to the MS method file, and submits the next sample to the queue. The PIE list contains both $\mathrm{m} / \mathrm{z}$ value and time window for each precursor ion, and is generated intelligently so that if an MS/MS is insufficient for identifying the peak of interest, it will be collected again near the top of the peak in the next run. Therefore, the strategy maximizes both quality and the number of unique MS/MS. When automated PIE was used to acquire LC-MS/MS data of an antibody tryptic digest and a soy hydrolysate sample, the number of identified ions increased by $52 \%$ and $93 \%$, respectively, compared with data acquired without using PIE.
\end{abstract}

Key words: Data-dependent acquisition, Tandem mass spectrometry, Intersample, Precursor ion exclusion, Proteomics, Metabolomics, Mixtures, Ion trap, Orbitrap

\section{Introduction}

T iquid chromatography-tandem mass spectrometry (LC$1 \mathrm{MS} / \mathrm{MS}$ ) has been increasingly used in the analysis of complex mixtures in many fields, including proteomics [1], metabolomics [2], full characterization of therapeutic proteins $[3,4]$, characterization of complex cell-culture raw materials and media, and investigation of protein conformation and dynamics by hydrogen/deuterium exchange [5, 6] and other labeling techniques [7, 8], etc. Many of these applications involve analysis of multiple samples with similar compositions.

Correspondence to: Zhongqi Zhang; e-mail: zzhang@amgen.com
In an LC-MS/MS experiment, the identity of an ion is usually determined from its determined mass and fragmentation pattern shown in its tandem mass spectrum (MS/MS). A data-dependent acquisition (DDA) technology is usually applied during data collection to select the precursor ions to perform tandem mass spectrometry (MS/MS). To identify a maximum number of ions, a "dynamic exclusion" principal has been employed during DDA. The dynamic exclusion technology temporarily puts the $\mathrm{m} / \mathrm{z}$ of an ion into an exclusion list for a set length of time after its MS/MS has been acquired. As a result, the instrument will not continuously acquire MS/MS of the same ion, but spend more time acquiring MS/MS of less abundant ions. The limitation of dynamic exclusion is that it works only within a 
single LC-MS/MS run. When multiple samples are analyzed in parallel, it is often the case that these samples share many common components, such as peptides from abundant housekeeping proteins in a proteomics experiment, common metabolites in a metabolomics experiment, and abundant unmodified peptides in the bottom-up protein characterization experiment. When an ion is identified from its MS/ MS in one run, it is not necessary anymore to collect its MS/MS in the remaining runs. Therefore, to collect a true maximum number of unique MS/MS, an "intersample data-dependent acquisition" (ISDDA) technique is necessary. An ISDDA technique involving precursor ion exclusion (PIE) has been developed by Wang and Li for proteomics experiments on a quadrupole time-of-flight instrument. The procedure was demonstrated to significantly increase the number of identified proteins [9]. This type of PIE procedure, although demonstrated to be beneficial, has not been widely adopted because the exclusion list needs to be generated and imported into the MS method file manually in most cases. For wide applications, the PIE list must be generated automatically after each run, and the MS method file for the next run must be modified automatically. Algorithms used for generation of the PIE list are important too. For example, when weak ions in early runs become strong in later runs, application of PIE increases the risk of missing the identification of these ions, which need to be addressed with more sophisticated algorithms for generating the PIE list.

An automatic ISDDA technology has been developed by Hoopmann et al. for post-analysis data acquisition (PAnDA), when a precursor ion list is used instead of an exclusion list to select the precursor ions intelligently for the next LC-MS/ MS run [10]. Using a precursor ion list offers a great advantage when a sample is analyzed multiple times, such as in many experimental settings when technical replicates are required. However, the requirement of PAnDA to analyze each sample multiple times limits its application in situations when replicate analysis is not necessary. Using an exclusion list, however, does not require multiple analyses of each sample.

In this work, an automated PIE technology is developed on Thermo Scientific ion-trap series of mass spectrometers. In this automated PIE technology, a precursor ion exclusion list is dynamically generated based on the data of previous LC-MS/MS runs. Background ions as well as precursor ions that have been selected for MS/MS in a previous run will be put into the exclusion list and, therefore, will not be selected for MS/MS in later runs. Although using an exclusion list was not favored by Hoopmann et al. attributable to the large size of the list [10], it served us well in most of our applications. This report describes in details how an exclusion list is generated, with a purpose of maximizing both the quality and number of unique MS/MS acquired during an LCMS/MS experiment.

\section{Method}

To identify a large number of components in a complex mixture, a "dynamic exclusion" technique is usually used to obtain a maximum number of unique MS/MS. Conventional dynamic exclusion has a problem that it only applies within one LC-MS/MS run. However, the concept of dynamic exclusion should also apply between samples. This idea can be accomplished by analyzing a data file to generate a precursor ion "exclusion list," and then adding the "exclusion list" into the MS method file, which will be used in later runs. This PIE approach has been described by Wang and Li for proteomics experiments [9]. In that work, Wang and $\mathrm{Li}$ evaluated four approaches to generate the PIE list and concluded that the best approach is to include both identified ions and nonidentifiable ions in the exclusion list. They also used a constant time window ( $\pm 150 \mathrm{~s})$ for the excluded ions, without examining the elution profile of the precursor peptide. Using a constant time window for generating the exclusion list is suitable for most proteomics experiments in which high-abundance peptides are usually less important than low-abundance peptides. However, in most other experiments, high-abundance species are always more important than low-abundance species. Therefore, one must not sacrifice the identification of high-abundance species for the identification of low-abundance species. Application of PIE using a constant time window was found to increase misidentification of high-abundance species. For example, the MS/MS of a very minor species in a control sample is performed and the ion is put into the exclusion list. However, the MS/MS does not generate a correct identification either because of poor MS/MS quality or interference from a stronger nearby ion. When the abundance of the species grows significantly in later runs, it never triggers an MS/MS because it is already in the exclusion list. To solve this and other similar problems, the following methodology is developed for generating the exclusion list. This methodology optimizes the best time window for precursor ion exclusion, aiming at maximizing the MS/MS quality and number of unique MS/MS at the same time. This automated routine is fully unattended, and is not restricted to any specific type of experiment. In this automated PIE approach, after each LCMS/MS run finishes, the raw data file is automatically analyzed to generate a list of precursor ions to be excluded, and this list is applied to the following run as an exclusion list for the selection of MS/MS precursor ions. The list contains information regarding the $\mathrm{m} / \mathrm{z}$ and elution time range of each precursor ion. The following shows how the exclusion list is generated from a data file after each LC-MS/MS run. A flow-diagram of the process is shown in Figure 1.

\section{Noise Level Determination}

When generating an exclusion list, the intensity of an MS signal often needs to be evaluated. Because signal intensities are usually instrument-dependent, we need an algorithm for 


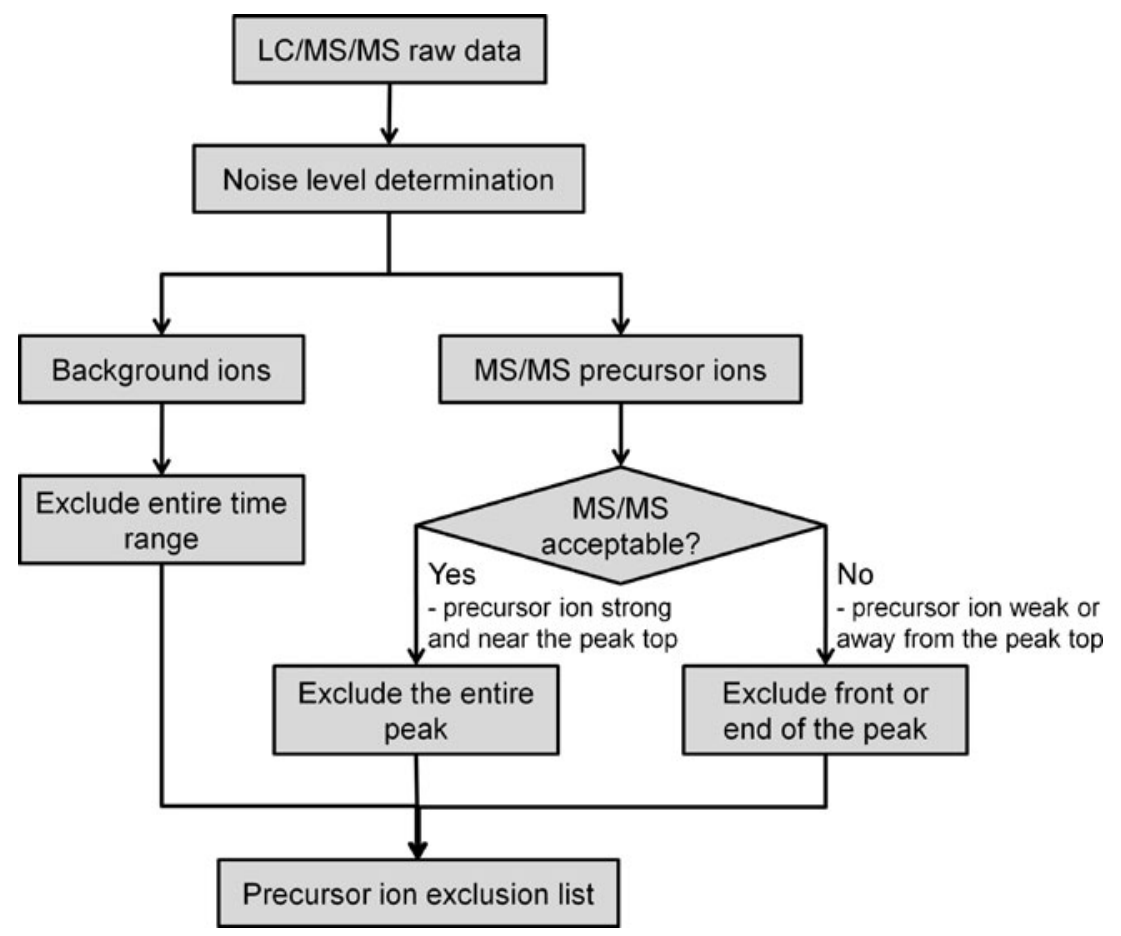

Figure 1. Flow-diagram of the process of generating an exclusion list from an LC-MS/MS data file. This exclusion list will be merged with the previous list and applied to the acquisition of the next LC-MS/MS run

a reasonable estimation of the noise level in the LC/MS run, and then the intensities of all signals can be evaluated relative to the noise level.

In an LC/MS run, signals appearing most frequently are usually noises. Therefore, the noise levels can be estimated from the distribution of all signals (including noise signals) in the entire run. To do that, all signals are put into bins according to their intensities (in logarithm scale) as shown in Figure 2. Assuming the intensity distribution of noise signals is Gaussian, and a desired maximum noise level should be above $98 \%$ (about two standard deviations) of all noise signals, the best estimate of maximum noise level should be the signal intensity at $13.5 \%$ of the maximum. However, considering the signal distribution is higher at the high-intensity side due to the presence of real signals, the signal intensity at $20 \%$ of the

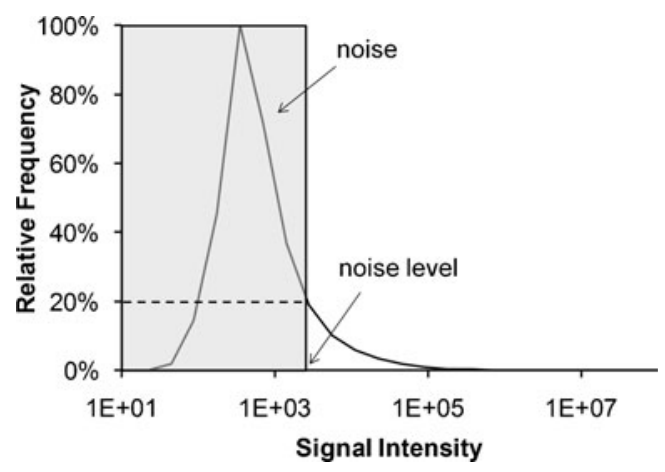

Figure 2. Distribution of all detected signal intensities in an $\mathrm{LC} / \mathrm{MS}$ run. The signal intensity at $20 \%$ of the maximum is used to represent the maximum noise level maximum is used to represent the maximum noise level (Figure 2). Indeed, a signal level at $20 \%$ of maximum usually matches the visually determined noise level.

\section{Exclusion of Background Ions}

The precursor ion exclusion list contains two types of ionsbackground ions and ions selected as MS/MS precursors. Background ions appear throughout the chromatogram; they should be excluded in later runs regardless of whether or not their MS/MS have been acquired. To obtain a list of background ions, all full-scan spectra in the LC-MS/MS run are summed together into a single spectrum. Because background ions are usually present in all scans, they typically have strong representation in this summed spectrum. The selected-ion chromatogram (SIC) of each ion above the noise level in this spectrum is then constructed [11] to determine whether the ion is a true background ion. If a chromatographic peak is not present in the SIC, or the width of the detected peak is too wide (wider than the userdefined maximum peak width) to be a real peak, the ion is considered to be a background ion. The $m / z$ of the ion is then added to the exclusion list, with exclusion time window covering the entire range of the chromatogram when this background ion is present.

\section{Exclusion of MS/MS Precursor Ions}

If the MS/MS of an ion has been acquired, the SIC of the ion is constructed. The SIC is smoothed using a Gaussian 
function with a width of one-third of a typical peak. From the SIC, the starting and ending time of the chromatographic peak is determined. The selected time window for ion exclusion depends on the intensity of the precursor ion, and the time when the MS/MS is performed. If the MS/MS is determined to be acceptable (will be discussed later), the ion is added to the exclusion list, with the time window covering the entire peak duration. If the quality of the MS/MS is determined not acceptable, either because the intensity of the precursor ion is too weak, or because the MS/MS is acquired too early, the ion is excluded only in the time window from the start of the peak to half of the peak maximum as shown in Figure 3 (top row). This approach ensures that a highquality MS/MS of the ion will be acquired near the top of the peak in the next run. The tail of the peak is not put into the exclusion list in this case because it will be excluded by regular intra-sample dynamic exclusion. Therefore, regular dynamic exclusion must be enabled in order for the automated PIE technique to work properly. The end of the peak is only put into the exclusion list when an MS/MS is triggered near the tail of the peak (Figure 3 bottom row). When an ion is added to the exclusion list, the $\mathrm{m} / \mathrm{z}$ of all its isotopes above the noise level are also added to the list.

Whether an MS/MS is acceptable or not is determined by (1) the absolute intensity of the precursor ion immediately before the MS/MS is acquired, and (2) the relative intensity

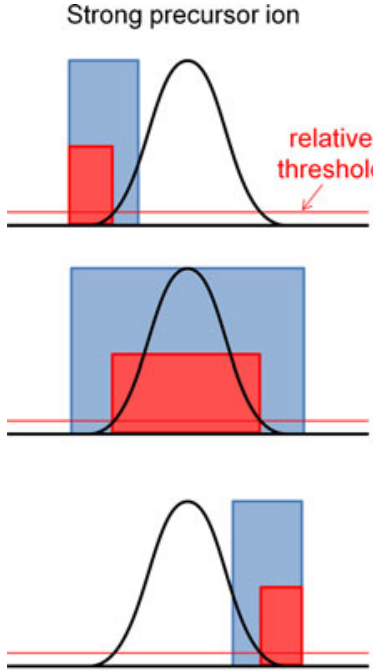

retention time $\rightarrow$
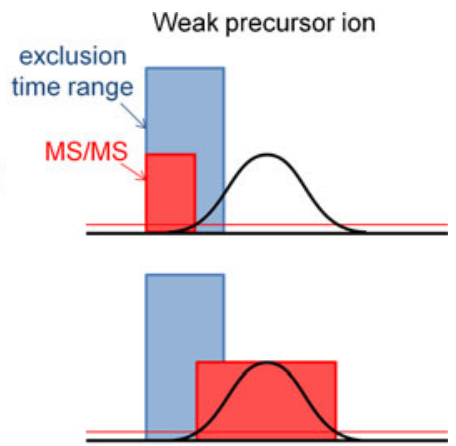

Figure 3. Determination of the exclusion time range for ions that has triggered MS/MS. The red regions are where MS/MS is acquired and the blue regions are the time range the ion will be excluded in the following run. Strong precursor ions and weak precursor ions are treated differently. The entire peak range is excluded only when the intensity of the precursor ion (immediately before the MS/MS is acquired) is higher than both the user-defined absolute and relative thresholds (left middle). When the precursor ion is below the relative intensity threshold (top and bottom rows) or the absolute intensity threshold (right column), only the front or end of the peak is excluded of the precursor ion as compared to the maximum intensity at the top of the SIC. Thresholds for both absolute and relative intensities are defined by the user. If the intensity of the precursor ion at the time of MS/MS is higher than both the absolute and relative thresholds, the MS/MS is considered to be acceptable and no more MS/MS is required for this ion in later runs (Figure 3 left middle). However, if either of the two thresholds is not reached, the MS/MS is not considered acceptable, in which case only the front or end part of the peak will be excluded so that a high-quality MS/MS will be acquired in the next run (Figure 3 top and bottom rows). The absolute intensity threshold ensures that a high-quality MS/MS will be acquired when a weak ion in earlier runs becomes stronger in later runs, and it can be set to zero for technical replicate runs. The relative intensity threshold ensures that at least one MS/MS of the ion will be acquired near the top of the SIC. When an MS/MS is performed too early or too late relative to the center of the chromatographic peak, it is not guaranteed that the MS/MS represents the peak of interest. Isomers are frequently observed in LC-MS/MS experiments (especially in metabolomics experiments), and these isomers frequently have similar retention times. Therefore, the identifications based on MS/MS acquired too early or too late are not always reliable. Incorrect conclusion may be made when the abundance of a species is calculated based on the identification of a lower-abundance isomers eluted slightly earlier or later than the species of interest. To have a reliable identification of a peak, the MS/MS must be performed near the top of the peak. The strategy applied here ensures that if the MS/MS is acquired too early or too late, it will be acquired again in the next run near the top of the peak.

The method described above for generating the exclusion list is based on the assumption that more abundant components are always more important than less abundant components. Therefore, one does not sacrifice the identification of a high-abundance component for the identification of a low-abundance component. In some cases, however, such as in a proteomic experiment where many housekeeping abundant proteins are not as interesting as lowerabundance proteins, the exclusion list can be generated with simpler algorithms, such as excluding the precursor ion over a user-specified constant time range whenever an MS/MS has been performed [9].

\section{Implementation}

Automated PIE is implemented in MassAnalyzer [11] through "Acquisition Control," an activeX control object provided with Thermo Scientific XCalibur. The routine implemented in MassAnalyzer is similar to the program PAnDA [10], in which a precursor ion list is generated and applied to later runs. PAnDA requires multiple analyses of a same sample, which is convenient and useful for experiments with technical replicates. Automated PIE implemented in MassAnalyzer, however, requires the samples to 
have similar composition but does not require each sample to be analyzed multiple times.

Owing to the large number of user-defined parameters used in automated PIE, for convenience of the users, MassAnalyzer is implemented in a way that the user only needs to provide an initial estimated chromatographic peak width (chromatographic peak width is defined in MassAnalyzer as twice of the peakwidth-at-half-height) for determining the parameters to smooth the SIC, and an "exclusion level," usually ranging from 1 to 10 , reflecting how aggressive the user likes automated PIE to be. All other parameters are derived from this exclusion level. For example, the relative threshold described in Figure 3 changes from $100 \%$ for exclusion level of 1 , to $10 \%$ for exclusion level of 10 . The absolute threshold is represented by a percentage of signals (above the noise level) that are over the threshold ( $0 \%$ to $90 \%$ for exclusion levels from 1 to 10 ). Keep in mind that automated PIE serves two purposes: (1) acquiring more unique MS/MS and (2) acquiring higher quality MS/MS. The exclusion level reflects a compromise between the two purposes. An exclusion level of 1 puts more weight on the quality of the acquired MS/MS, and a level of 10 puts more weight on the number of unique MS/MS acquired. The duration of regular intra-sample dynamic exclusion (a parameter in an XCalibur MS method) is also automatically set by MassAnalyzer to be half of the peak width (level=1) to the full peak width (level=10). With exclusion level above 10 (both absolute and relative thresholds are low), the method of generating the exclusion list approaches the constant time window method describe by Wang and Li [9], except that the method described here gives better-defined time windows by examining the elution profiles (SIC).

To collect LC-MS/MS data using automated PIE on MassAnalyzer, the user first sets up the MS method file and sequence file using XCalibur, and then loads the sequence file into MassAnalyzer. When running the sequence file, MassAnalyzer submits one sample at a time to the Xcalibur sequence queue. After data acquisition of the first sample finishes, MassAnalyzer analyzes the data file automatically to generate the exclusion list, adds the exclusion list to the global reject mass list in the MS method file, then submits the next sample using the modified MS method. When the data acquisition of the second sample finishes, MassAnalyzer generates an exclusion list from the second data file, merges the list to the previous exclusion list, and adds the new list to the MS method file. This process continues for the rest of the sequence.

Automated PIE requires the retention time of each component to be stable between LC-MS/MS runs. Shift of retention time deteriorates the effect of the technique. To minimize the problem, instead of merging the exclusion lists of all previous runs together, the list is occasionally refreshed (the previous list discarded). The frequency of this refreshment is a user-defined parameter, which is set in MassAnalyzer to refresh after every $n$ runs, where $n$ is the user-defined exclusion level. The refreshment also occurs automatically when MassAnalyzer detects a significant retention time shift between the previous two runs, or a significant decrease in the number of acquired $\mathrm{MS} / \mathrm{MS}$, indicating that all available precursor ions are excluded at certain time points.

\section{Experimental}

For testing automated PIE, an IgG1 monoclonal antibody (Amgen, Thousand Oaks, CA, USA) was reduced and carboxymethylated, followed by digestion with trypsin (Roche, Indianapolis, IN, USA), using a procedure similar to previously described [12]. The digest was analyzed on a Thermo Scientific LTQ-Orbitrap high-resolution mass spectrometer connected to an Agilent (Santa Clara, CA, USA) 1290 Infinity LC system. A Waters (Milford, MA, USA) BEH $300 \mathrm{C} 18$ reversed-phase column $(1.7 \mu$ particle, $150 \times 2.1 \mathrm{~mm})$ was used for the separation, followed by electrospray ionization. Peptides were eluted with a linear acetonitrile gradient ( $1 \%$ to $40 \%$ in $44 \mathrm{~min}$, followed by column washing with $40 \%$ to $99 \%$ acetonitrile) at a flow rate of $0.2 \mathrm{~mL} / \mathrm{min}$, with $0.02 \%$ trifluoroacetic acid (TFA) in the mobile phase. About $16 \mu \mathrm{g}$ of protein digest was injected for each analysis.

In another experiment, a soy hydrolysate (Kerry BioScience, Norwich, NY, USA) sample was diluted into $1 \mathrm{mg} /$ $\mathrm{mL}$ with $10 \mathrm{mM} \mathrm{HCl}$, followed by analysis on a Thermo Scientific LTQ-Orbitrap high-resolution mass spectrometer connected to an Agilent 1200 LC system. A Waters BEH 130 C18 reversed-phase column $(1.7 \mu$ particle, $100 \times 2.1 \mathrm{~mm})$ was used for the separation, followed by electrospray ionization. Components were eluted with a linear acetonitrile gradient ( $0 \%$ to $30 \%$ in $19 \mathrm{~min}$, followed by column washing using 30 $99 \%$ acetonitrile) at a flow rate of $0.325 \mathrm{~mL} / \mathrm{min}$, with $0.2 \%$ heptafluorobutyric acid (HFBA) in the mobile phase. About $10 \mu \mathrm{L}$ of sample was injected for each analysis.

In both experiments, each sample was analyzed by LCMS/MS five times consecutively. The first three runs were performed without PIE, run 4 was performed with the PIE list generated from run 3, and run 5 was performed with the PIE list generated from runs 3 and 4 . The MS method was set up to collect one full scan in the high-resolution Orbitrap (resolution $=60,000$ at $\mathrm{m} / \mathrm{z} 400$ ), followed by two collisioninduced dissociation (CID) MS/MS scans in the linear trap. Regular intra-sample dynamic exclusion was enabled with exclusion duration set at about three-quarters of a typical peak width (12 s for the protein digests and $11 \mathrm{~s}$ for the soy hydrolysate sample). For data collected with automated PIE, a reject mass width of $\pm 0.02(\mathrm{~m} / \mathrm{z}$ scale $)$ was used. Data acquisition was controlled by MassAnalyzer. An exclusion level of 10 was used for the protein digest and 5 used for the soy hydrolysate sample.

\section{Data Analysis}

For the antibody tryptic digest, data from runs 1-3 and runs 3-5 were analyzed separately by MassAnalyzer, in which peak detection and retention-time alignment [13] were performed, followed by peptide identification by comparing 
experimental MS/MS to the theoretically predicted MS/MS [14-17]. The peptide search space included specific and half-specific tryptic peptides of the heavy chain and the light chain of the IgG1 molecule, porcine trypsin, as well as the reverse sequences of the three proteins. Search by MassAnalyzer also included peptides with specified and unrestricted modifications [11, 17], glycopeptides [16], and peptides with amino-acid substitutions.

Similarly, for the soy hydrolysate sample, data from runs 1-3 and runs 3-5 were analyzed separately by MassAnalyzer, in which peak detection and retention-time alignment were performed, followed by component identification by correlating experimental MS/MS to the reference MS/MS in a custom-built MS/MS spectral library. The library contained primarily entries in the NIST08 MS/MS library (National Institute of Standard and Technology) and an NIST dipeptide library (kindly contributed by Stephan Stein/ Sara Yang from NIST), with additional entries from this laboratory. De novo sequencing [18] was used to identify peptides three residues or longer.

\section{Results and Discussion}

A tryptic digest of an IgG1 antibody was analyzed three times without PIE, followed by an automated PIE run using the exclusion list generated from the third run, and another automated PIE run using the exclusion list generated from the third and fourth runs. Data acquisition was controlled by MassAnalyzer, and an exclusion level of 10 (relative threshold $=10 \%$; absolute threshold $=$ top $90 \%$ of all signals above the noise level) was used for the two automated PIE runs. The exclusion list for the fourth run contained 9523 entries and the list for the fifth run contained 10,954 entries.

The above experiment generated two datasets that can be used for direct comparison of LC-MS/MS data acquired with and without using automated PIE. The first dataset contained runs 1-3, none of which used any exclusion list for acquisition. The second dataset contained runs 3-5 (with run 3 shared between the two datasets), which reflected exactly how automated PIE should be performed for three consecutive runs. The two datasets were processed separately by MassAnalyzer. It has been demonstrated that for identification of modified and unmodified peptides from a purified protein, the search algorithm provided by MassAnalyzer is more advantageous than conventional search algorithm designed for proteomics purposes [11]. The false-discovery rates (FDR) calculated here are not accurate due to lack of statistics from the small search space. Therefore, effort is not applied to make the FDRs in the two data sets exactly the same. Instead, a confidence threshold of 0.8 was used for all peptide identification [11]. Figure 4 shows the Venn diagrams of the number of peptides identified in each run in the two sets of data. It is seen that although the sample was analyzed three times in both cases, 704 more peptide ions (52\%) were identified when automated PIE was applied. As expected, because a same
Without PIE

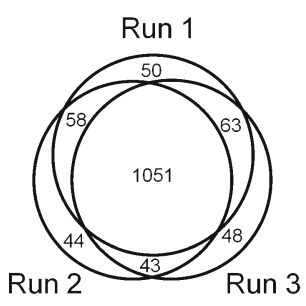

Total ions identified

- After 1 run: 1222

- After 2 runs: 1309

- After 3 runs: 1357

FDR $=0.2 \%$
With automated PIE

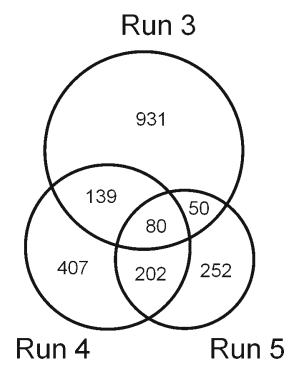

Total ions identified

- After 1 run: 1200

- After 2 runs: 1809

-After 3 runs: 2061

$\mathrm{FDR}=0.6 \%$
Figure 4. Venn diagram showing the number of peptide ions identified in three LC-MS/MS analyses of a tryptic digest of an antibody with and without using automated PIE. The false-discovery rate (FDR) of peptide identification is estimated based on the reverse sequence hits

MS method was used for runs 1-3, there was a large overlap of identified peptides among these three runs. When automated PIE was applied, however, the number of overlapping identification was greatly reduced. It was also interesting to note that fewer and fewer peptides were identified in later runs with automated PIE. This phenomenon was caused by the exclusion of all strong ions in later runs, therefore limiting MS/ MS experiments to only weak ions, which were less likely to be confidently identified due to poor spectral quality and interferences from other ions co-isolated with the precursor ions. The fact that the number of peptides identified in each run decreased with the application of automated PIE suggested that the exclusion level of 10 was not necessary for a sample with this level of complexity; a decreased exclusion level may potentially increase the MS/MS quality without significant sacrifice of the unique number of MS/MS.

Peptide mapping data collected on a high-resolution LC-MS/MS system, combined with advanced data analysis algorithms, makes it possible to identify and quantify therapeu-

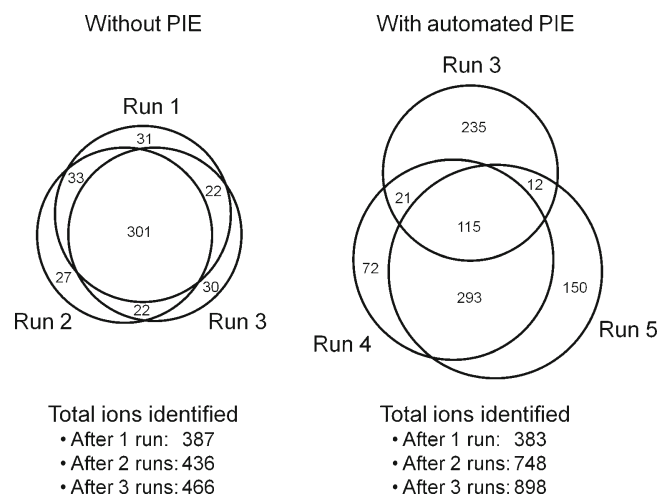

Figure 5. Venn diagram showing the number of components identified in three LC-MS/MS analyses of a soy hydrolysate sample with and without using automated PIE 
tic protein isoforms on a large scale [11]. Application of the automated PIE technology further increases the number of modifications that can be identified and quantified in a single experiment. For example, the automated PIE technology has been proven to be very useful for detecting and identifying extremely small amount of protein isoforms $(0.001 \% \sim 0.1 \%)$, such as those caused by aminoacid substitutions (data not shown).

To further demonstrate the capability of automated PIE, a soy hydrolysate sample was analyzed similarly. Soy hydrolysate contains a large number of components, including amino acids, short peptides, carbohydrates, B vitamins, and other nutrients, and is widely used as a source of nutrients for cell culture media. The sample was again analyzed three times without automated PIE, followed by an automated PIE run using an exclusion list generated from the third run, and another automated PIE run using exclusion list from the third and fourth runs. Data acquisition was controlled by MassAnalyzer and an exclusion level of 5 (relative threshold=20\%; absolute threshold $=$ top $10 \%$ of all signals above the noise level) was used for the two automated PIE runs. The exclusion list for the fourth run contained $2018 \mathrm{~m} / \mathrm{z}$ entries and the list for the fifth run contained 3588 entries. The main reason for the decreased number of excluded ions in this experiment was the decreased number of isotope peaks for small-molecule components.

Similarly, data from runs $1-3$ and runs 3-5 were processed separately by MassAnalyzer. Figure 5 shows the Venn diagrams of number of components identified in each run in the two sets of data. Although the sample was analyzed three times in both cases, 432 more components ( $93 \%)$ were identified when automated PIE was applied. As expected, because the same MS method was used for runs $1-3$, there was a large overlap of identified components among these three runs. When automated PIE was applied, however, the number of overlapping identification was greatly reduced. It was interesting to note that in this case, more and more components were identified in later runs when automated PIE was applied. The reason was that an exclusion level of 5 represented a good compromise between the MS/MS quality and number of unique MS/MS acquired. Applying automated PIE not only increased the number of unique MS/MS, but it also improved the MS/MS quality by acquiring MS/MS near the top of the peak, therefore improving the chance of identification. Run 4 and run 5 spent most time acquiring MS/MS of weaker ions, and weaker ions were usually not fully excluded with an exclusion level of 5 (Figure 3 right) to ensure good MS/MS quality in later runs. As a result, a much larger overlap of identified components was observed between run 4 and run 5 . The ideal selection of exclusion level and, therefore, parameters used to generate the exclusion lists, depends on the complexity of the samples, the interest in weaker ions compared with stronger ions, the similarity of the samples, and the number of samples analyzed together, etc.

Please note that the multiple analyses of a same sample are shown here for the purpose of demonstrating the benefits of automated PIE. In practical applications when multiple samples are analyzed, replicate analyses of each sample, although beneficial, are not required. The impact of this technique, however, is highly dependent on the similarity of samples analyzed. The more similar the samples are, the more effective the technique will be. Therefore, the results shown here represent the best-case scenario. As the worstcase scenario, the impact of the technique will be minimal when the samples are very different.

Hoopmann et al. has found using an exclusion list generated a list too large for their applications [10]. We found, however, the exclusion list serves the purpose for most of our applications, in which the size of the list is rarely more than 50,000. Automated PIE has been routinely used in our laboratories for various LC-MS/MS applications including identification of amino acid substitutions, post-translational and process-induced modifications of therapeutic proteins, characterization of complex cell-culture raw materials and media, as well as metabolomics and some proteomics experiments.

\section{Acknowledgment}

The author is indebted to Dr. Michael MacCoss and Dr. Michael Hoopmann of the University of Washington for their help to get the author started in using the activeX control for data acquisition. The author also thanks Stephan Stein and Sara Yang at the National Institute of Standard and Technology for sharing their dipeptide MS/MS library, Jason Richardson and Bhavana Shah of Amgen for collecting data to test the algorithms, and Pavel Bondarenko, Jason Richardson, and Da Ren for helpful discussions during the development of the program. This work was funded by Amgen Inc.

\section{References}

1. Yates, J.R., Ruse, C.I., Nakorchevsky, A.: Proteomics by mass spectrometry: Approaches, advances, and applications. Annu. Rev. Biomed. Eng. 11, 49-79 (2009)

2. Bedair, M., Sumner, L.W.: Current and emerging mass-spectrometry technologies for metabolomics. Trac-Trends Anal. Chem. 27, 238-250 (2008)

3. Srebalus Barnes, C.A., Lim, A.: Applications of mass spectrometry for the structural characterization of recombinant protein pharmaceuticals. Mass Spectrom. Rev. 26, 370-388 (2007)

4. Zhang, Z., Pan, H., Chen, X.: Mass spectrometry for structural characterization of therapeutic antibodies. Mass Spectrom. Rev. 28, 147-176 (2009)

5. Zhang, Z., Smith, D.L.: Determination of amide hydrogen exchange by mass spectrometry: a new tool for protein structure elucidation. Prot. Sci. 2, 522-531 (1993)

6. Engen, J.R.: Analysis of protein conformation and dynamics by hydrogen/deuterium exchange MS. Anal. Chem. 81, 7870-7875 (2009)

7. Xu, G., Chance, M.R.: Hydroxyl radical-mediated modification of proteins as probes for structural proteomics. Chem. Rev. 107, 35143543 (2007)

8. Novak, P., Giannakopulos, A.E.: Chemical cross-linking and mass spectrometry as structure determination tools. Eur. J. Mass Spectrom. 13, 105-113 (2007)

9. Wang, N., Li, L.: Exploring the precursor ion exclusion feature of liquid chromatography-electrospray ionization quadrupole time-of-flight mass spectrometry for improving protein identification in shotgun proteome analysis. Anal. Chem. 80, 4696-4710 (2008) 
10. Hoopmann, M.R., Merrihew, G.E., von Haller, P.D., MacCoss, M.J.: Post-analysis data acquisition for the iterative $\mathrm{ms} / \mathrm{ms}$ sampling of proteomics mixtures. J. Proteome Res. 8, 1870-1875 (2009)

11. Zhang, Z.: Large-scale identification and quantification of covalent modifications in therapeutic proteins. Anal. Chem. 81, 8354-8364 (2009)

12. Ren, D., Pipes, G.D., Liu, D., Shih, L.-Y., Nichols, A.C., Treuheit, M.J., Brems, D.N., Bondarenko, P.V.: An improved trypsin digestion method minimizes digestion-induced modifications on proteins. Anal. Biochem. 392, 12-21 (2009)

13. Zhang, Z: Retention time alignment of LC/MS data by a divideand-conquer algorithm. J. Am. Soc. Mass Spectrom. 23, 764-772 (2012)
14. Zhang, Z.: Prediction of low-energy collision-induced dissociation spectra of peptides. Anal. Chem. 76, 3908-3922 (2004)

15. Zhang, Z.: Prediction of low-energy collision-induced dissociation spectra of peptides with three or more charges. Anal. Chem. 77, 6364-6373 (2005)

16. Zhang, Z., Shah, B.: Prediction of collision-induced dissociation spectra of common n-glycopeptides for glycoform identification. Anal. Chem. 82, 10194-10202 (2010)

17. Zhang, Z.: Prediction of collision-induced-dissociation spectra of peptides with post-translational or process-induced modifications. Anal. Chem. 83, 8642-8651 (2011)

18. Zhang, Z.: De novo peptide sequencing based on a divide-and-conquer algorithm and peptide tandem spectrum simulation. Anal. Chem. 76, 6374-6383 (2004) 